\title{
Coordinación docente e interdisciplinariedad para la adquisición de competencias en el Grado de Educación Primaria e Infantil: Percepciones de alumnado y profesorado
}

\author{
Teaching and interdisciplinary coordination for the acquisition of competencies in \\ the Primary Education Degree: Students and faculty perceptions
}

\author{
María José Arroyo González \\ e-mail: mjarroyo@pdg.uva.es \\ Universidad de Valladolid. España \\ Ruth Pinedo González \\ ruth.pinedo@psi.uva.es \\ Universidad de Valladolid. España \\ Myriam de la Iglesia Gutiérrez (iD) \\ mdelaig@psi.uva.es \\ Universidad de Valladolid. España
}

\section{Resumen}

La coordinación docente es fundamental para conseguir el cambio de paradigma que el Espacio Europeo de Educación Superior nos demanda. El trabajo coordinado y la realización de actividades interdisciplinares favorecen la adquisición de competencias profesionales necesarias para la vida laboral futura de nuestro alumnado. En este estudio presentamos los datos relativos a la percepción que alumnado y profesorado de la Universidad de Valladolid tiene tras la realización de una práctica interdisciplinar entre dos asignaturas del Grado de Educación. Se ha utilizado una metodología mixta con un diseño transversal. Se han diseñado dos instrumentos ad hoc para la recogida de datos de los estudiantes y del profesorado participante. Resultados: En relación a la percepción de los estudiantes, se ha valorado muy positivamente la práctica interdisciplinar por ser considerada útil y que favorece la comprensión de los contenidos de las asignaturas implicadas. Además se ha considerado de forma muy positiva el seguimiento que se ha realizado de los trabajos de investigación, la documentación y organización de la práctica. Se ha considerado de forma menos positiva las labores de coordinación. En relación a la percepción del profesorado se ha considerado positivo el aprendizaje mutuo derivado de la coordinación pero se ha destacado como aspecto negativo el aumento de trabajo y el esfuerzo que suponen este tipo de prácticas. En general es muy interesante y productivo llevar a cabo este tipo de prácticas interdisciplinares, pero deben ser cuidadosamente diseñadas y planificadas.

Palabras clave: universidad; innovación educativa; desarrollo profesional; educación superior; profesorado.

\begin{abstract}
Teacher coordination is fundamental to achieve the new paradigm that the European Higher Education Area demands. The coordination and realization of interdisciplinary activities facilitate the acquisition of professional competencies necessary for our students' future performance in the workplace. In this study we present the data concerning the perception that students and teachers of the University of Valladolid have after the realization of an interdisciplinary initiative between two subjects taught as part of the Degree in Education. A mixed methodology has been used with a cross-sectional design. Two ad hoc instruments have been designed for the collection of data from students and teachers. Results: Regarding student perception, the interdisciplinary practice sessions have been highly valued for being considered useful and helpful in understanding the contents of the subjects taught. In addition, the follow-up done of the research work, documentation and organization of the sessions has also been considered as highly positively. The coordination of teachers has been less positively considered. With regards to the perception of the professors the mutual learnings derived from the coordination of the activity have been considered positive, but the increase of work and effort that this type of sessions require has been highlighted as a negative aspect. In general, it is very interesting and productive to carry out this type of interdisciplinary practice sessions, but they must be carefully designed and planned. New technologies can be a useful resource to help teacher coordination.
\end{abstract}

Key words: university; education innovation; professional development; higher education: teachers.

Recibido / Received: 18-02-19 | Aceptado / Accepted:06-04-19 | Publicación en línea / Published online: 16-09-19

Cómo referenciar este artículo / How to reference this article:

Arroyo, M. J., Pinedo, R., \& Iglesia, M. de la (2020). Coordinación docente e interdisciplinariedad para la adquisición de competencias en el Grado de Educación Primaria e Infantil: Percepciones de alumnado y profesorado. Tendencias Pedagógicas, 35, 2020, pp.102-117. doi: 10.15366/tp2020.35.009 


\section{Introducción}

\subsection{El EEES: nuevas posibilidades en el proceso de enseñanza-aprendizaje}

El Espacio Europeo de Educación Superior (EEES), más allá del cambio en la organización y en el diseño e implementación de los títulos de grado, ha supuesto la posibilidad de reflexionar sobre cómo queremos que sea el proceso de enseñanza-aprendizaje en la universidad, tanto para el discente como para el docente. El cambio fundamental pasa por centrarse en qué y cómo está aprendiendo el alumnado y no en lo que el profesorado enseña. Este proceso de cambio abre la puerta a la innovación pedagógica en el ámbito universitario, más allá del cambio en las técnicas docentes, se impone un cambio en la mentalidad y afrontar nuevos retos y formas de trabajo. Se hace imprescindible la coordinación del profesorado y superar el individualismo tradicional de la educación superior. Durante las últimas dos décadas se ha enfatizado la importancia de un cambio de paradigma en el ámbito educativo que posibilitase el paso de una educación centrada en la enseñanza a otra centrada en el aprendizaje (Scott, 2015). En este nuevo espacio de aprendizaje surge un nuevo concepto: las competencias. Según Palmer, Montaño y Palou (2009) las competencias son la capacidad aprendida para realizar de manera adecuada una tarea, función o rol relacionados con el ámbito particular de trabajo que integran conocimientos, habilidades y actitudes. Son las capacidades finales que nuestro alumnado debe conseguir, lo que debe saber para llevar a cabo su futura práctica diaria (Carreras y Perrenoud, 2005). El objetivo de la formación universitaria debe ser formar profesionales que sean capaces de analizar los problemas cotidianos, poseer una visión comprehensiva de los fenómenos y trabajar de forma cooperativa en la resolución de los problemas que en su vida profesional pueden acontecer (Martínez y Viader, 2008).

\section{La formación del alumnado: aprendizaje de competencias}

Como venimos analizando, en la actualidad los esfuerzos del profesorado deben ir encaminados a saber qué están aprendiendo nuestros estudiantes de todo lo que se les enseña, qué relaciones están estableciendo entre los contenidos y, en consecuencia, qué habilidades están desarrollando.

El nuevo espacio de aprendizaje requiere de una mayor implicación del alumnado y del profesorado en el proceso de aprendizaje, así como la necesidad de aprender a trabajar cooperativa y colaborativamente (Johnson y Johnson, 2014; Scott, 2015). Se plantea el aprendizaje para el desarrollo de competencias, y en consecuencia, el trabajo interdisciplinar. Cuadrado, Ruiz y Coca (2009) afirman que los aprendizajes interdisciplinares basados en el trabajo colaborativo y la coordinación del profesorado nos permiten trabajar dos logros: conseguir un aprendizaje basado en la adquisición de competencias del alumnado y el desarrollo profesional de los docentes.

El documento DESECO (OCDE, 2003) recoge también la importancia del concepto de competencias, que se definen como un conocimiento activo, aplicado, no solamente acumulado, que atiende a las implicaciones prácticas que pueden tener estos conocimientos. Supone la superación del aprendizaje tradicional y plantea cambios en la concepción de las titulaciones y también a la hora de planificar e implementar las materias.

Uno de los primeros desafíos está en la coordinación del profesorado, y se presenta como un requisito imprescindible a la hora de facilitar la adquisición de competencias por parte del alumnado, así como el diseño de instrumentos que permitan evaluar de manera fiable el proceso de aprendizaje de nuestros alumnos (López-Pastor y Pérez-Puello, 2017).

3. Coordinación del profesorado universitario y trabajo colaborativo como posibilidad: una propuesta interdisciplinar

El nuevo reto al que el profesorado universitario se enfrenta pasa por ser un profesor con formación docente, que no solamente esté especializado en su materia, sino que sepa cómo contribuir a que su alumnado aprenda (Jiménez y Coronil, 2018). Como apunta Zabalza (2006), la coordinación del profesorado se basa en una estructura organizativa de tipo horizontal y vertical, basada en la 
voluntariedad y el interés en participar en actividades conjuntas para promover redes sociales de aprendizaje. La coordinación entre docentes supone dar a conocer al otro dónde estamos, es decir, compartir nuestra posición en términos de programación, metodologías, etc. En consecuencia, la coordinación del profesorado supone unificar los puntos de vista sobre el acto educativo. Es desde este lugar donde tienen cabida los proyectos o prácticas interdisciplinares, que cumplen una función importante de cara a promover el aprendizaje de y por competencias en los nuevos grados (Jiménez y Coronil, 2018; Krichesky y Murillo, 2015).

El trabajo colaborativo en el ámbito educativo ha sido ampliamente investigado. Así encontramos diversas líneas, desde las primeras que asumen la colaboración como factor de cambio educativo (Hargreaves y Shirley, 2012), pero también como componente clave del aprendizaje organizacional (Gairín, 2000; Senge, 2002) y como estrategia de mejora de los centros escolares (McLaughlin y Talbert, 2006). Como apuntan Krichesky y Murillo (2015), lo importante es reconocer cuáles son las modalidades de trabajo colectivo que impulsan la innovación y qué tipo de intercambios promueven la capacidad de mejora del profesorado.

El EESS implica cambios muy significativos en el ámbito docente, donde la coordinación, colaboración y cooperación entre los docentes pasa a ser uno de los aspectos principales (Scott, 2015). La coordinación docente debe plantearse desde la coordinación de los objetivos de aprendizaje, los contenidos, los métodos docentes, los calendarios de actividades y, por supuesto, la evaluación (Torrego y Ruiz, 2011).

Gallego, Redondo, Lorente y Benedito (2011) analizan los principales obstáculos que dificultan la posibilidad de alcanzar un nivel óptimo de coordinación en el profesorado y entre ellos se señalan el individualismo de los profesores universitarios, las reticencias a exponer sus métodos docentes y sentirse evaluados, y la actual política universitaria que no incentiva ni reconoce la coordinación entre docentes. En este sentido, la coordinación docente supone una gran inversión en tiempo y esfuerzo que no está reconocida en términos económicos o de carrera universitaria.

\section{El trabajo colaborativo como posibilidad: una propuesta interdisciplinar}

El primer requisito para el trabajo colaborativo en la universidad pasa por una buena coordinación, tanto vertical como horizontal (Zabalza 2006) que permita a los profesores superar la parcelación de los contenidos y relacionar unas materias con otras (Pou, 2009). La interdisciplinariedad entre las distintas materias se plantea como una manera óptima de aprender, ya que permite a los discentes comprender los problemas reales de su campo profesional de una manera global, pero también presenta ventajas para los docentes, ya que les ayuda a mejorar su desarrollo profesional. Todo ello muy relacionado con un proceso de investigación-acción donde el docente investiga sobre su propia práctica, realizando modificaciones para su mejora.

Como apuntan diversos autores (Donmoyer, Yennie-Donmoyer y Galloway, 2012; Sebastian y Allensworth, 2012; Vescio, Ross y Adams, 2008) en sus investigaciones, la colaboración entre docentes es una condición esencial para impulsar procesos de innovación y mejora en los centros educativos, pero como advierten Little y Horn (2007) no cualquier práctica colaborativa supone procesos innovadores en sí mismos.

Es necesaria la colaboración y cooperación entre docentes si queremos que los alumnos adquieran las competencias finales. Senge (2002) apuesta por el trabajo en equipo en las organizaciones modernas. Esta idea, como vemos, está en contraposición con las culturas organizativas existentes en nuestras universidades, caracterizadas por la prevalencia de identidades individuales, que se manifiestan en la planificación y la articulación de estrategias de desarrollo individual.

Existen experiencias previas de mejora de la coordinación entre el profesorado. Así, encontramos algunas en diferentes asignaturas de una misma titulación (Learreta, 2006); o profesores que imparten la misma materia en distintas titulaciones (Sánchez-Cantalejo, Rodríguez, Sáez, Velasco y Fernández, 2001), aunque también puede darse entre profesores de distintas áreas de conocimiento y titulaciones de impartición. Entre los beneficios que estas experiencias aportan, vemos cómo el trabajo en equipo es más eficiente que las acciones individuales, permite a los docentes una reflexión sobre los problemas comunes, las fortalezas y las debilidades, y determinar unos criterios comunes que guíen la actuación. Además, facilitan un ambiente que desarrolla la motivación del alumnado y, tal y como apuntan Smith y Scott (1990), promueven mejores niveles de autoestima en el profesorado, así como el 
desarrollo de las competencias comunicativas e interpersonales y el sentimiento de pertenencia a la institución educativa.

Otra experiencia que hace interesantes aportaciones en este campo son las Lesson Studies. Se trata de una metodología de investigación y mejora de la práctica educativa originaria y con una amplia tradición en Japón y con gran repercusión en Norteamérica y, recientemente, en Europa. El objetivo principal es mejorar la práctica educativa (Simón, Echeita y Sandoval, 2018).

La Lesson Study es una metodología de investigación que pretende mejorar la práctica educativa. Engloba un conjunto de prácticas, hábitos mentales, relaciones personales y herramientas que ayudan a los docentes a trabajar en colaboración y a mejorar su práctica. Por tanto, ayuda a mejorar la práctica educativa y la investigación. Los profesores reformulan la metodología que utilizan, los contenidos que enseñan, el aprendizaje del alumnado... En definitiva, un trabajo colaborativo y crítico del proceso de enseñanza-aprendizaje (Pérez y Soto, 2011).

Por su parte, Krichesky y Murillo (2015) explican cómo en el aprendizaje colaborativo entre los docentes la colaboración puede adoptar distintos matices y concretarse a través de diferentes prácticas como: (1) La coordinación; (2) El desarrollo conjunto de actividades y de esfuerzos para conseguir un trabajo o proyecto final; y (3) La resolución de problemas que aparecen en la práctica docente.

5. Aprendizaje orientado a proyectos y aprendizaje colaborativo en las prácticas interdisciplinares

Para la realización de este tipo de prácticas se recomienda el uso de metodologías activas, como el Aprendizaje orientado a Proyectos (AOP) y el Aprendizaje colaborativo (AC), ya que ambas ayudan al desarrollo de competencias y de conocimiento de una manera global e interdisciplinar (Navarro y López, 2019; Pinedo, Caballero y Fernández, 2016; Berzosa y Arroyo, 2014). Mientras que en el AOP el alumnado toma un papel más activo en la construcción de su aprendizaje, mayor compromiso y responsabilidad, así como un aprendizaje más profundo (Morales, 2007); en el AC se pone en marcha el trabajo en equipo del alumnado, el cual favorece el desarrollo de habilidades interpersonales e intelectuales, la gestión de la responsabilidad y la autonomía en el aprendizaje y la motivación (Aranda, 2015; Benito y Cruz, 2005; Durall y Leionen, 2014; Jaranta, 2014; Johnson, Johnson y Holubec, 1999; Johnson y Johnson, 2014; Navarro y López, 2019; Peñaloza, 2017).

En resumen, este trabajo se plantea los siguientes objetivos: (1) Conocer el interés suscitado en el alumnado y el profesorado por la realización de una práctica interdisciplinar; (2) Valorar cómo perciben el alumnado y el profesorado la coordinación docente en la realización de dicha práctica interdisciplinar; (3) Analizar cómo perciben el alumnado y el profesorado el desarrollo de dicha práctica interdisciplinar; Y (4) Reflexionar sobre la viabilidad de la realización de prácticas interdisciplinares en los estudios de grado.

\section{Método}

\subsection{Participantes}

La muestra está formada por 75 estudiantes de $2^{\circ}$ curso del Grado de Educación de la Universidad de Valladolid. La muestra fue seleccionada mediante un muestreo no probabilístico por conveniencia. La edad media de los participantes es de 21.27 años (d.t. = 3.35), con un mínimo de 19 años y un máximo de 42 años. El $71.6 \%$ de la muestra son mujeres. La muestra analizada se encuentra matriculada en el Grado de Educación Primaria (52.7 \%) o en el Programa de estudios conjuntos del Grado en Educación Infantil y Primaria (47.3 \%). También fueron participantes de este estudio el profesorado que diseñó y llevó a cabo esta investigación, que fueron tres mujeres con una edad media de 39 años (d.t.=1.6). La investigación se desarrolló durante el curso 2017-2018. 


\subsection{Descripción de la experiencia}

En este artículo se presenta una de las experiencias que se están llevando a cabo en coordinación docente e interdisciplinariedad en la Universidad de Valladolid. Esta experiencia implica dos asignaturas del módulo de formación básica del Grado de Educación Primaria y el programa de estudios conjuntos del Grado de Educación Infantil y Primaria y a los docentes responsables de las mismas. Las asignaturas son «Métodos de Investigación e Innovación en Educación» y «Fundamentos Psicopedagógicos de la Atención a la Diversidad», ambas de $2^{\circ}$ curso.

La experiencia docente de años anteriores nos decía que los estudiantes tienen dificultades para encontrar el sentido y la vinculación con la práctica profesional de la asignatura de «Métodos de investigación e Innovación en Educación», por ello nos planteamos vincular esta materia a los contenidos de otra, en este caso la de «Fundamentos Psicopedagógicos de Atención a la Diversidad». Creíamos que el hecho de realizar su Proyecto de investigación sobre un tema concreto de otra asignatura contribuiría a facilitar la comprensión de los contenidos de ambas disciplinas, a la vez que proporcionaría conocimientos más globales y aplicados.

La experiencia que presentamos consistió en diseñar un proyecto o práctica interdisciplinar utilizando metodologías activas como el aprendizaje basado en proyectos (ABP) y el aprendizaje cooperativo $(\mathrm{AC})$. Este tipo de prácticas interdisciplinares tienen como objetivo favorecer un conocimiento más global de los contenidos del grado y desarrollar de forma más adecuada las competencias comunes de las asignaturas implicadas y del grado.

El alumnado matriculado en ambas asignaturas y dividido en grupos de 3 o 4 personas ha realizado una práctica interdisciplinar conjunta en la que se han incluido contenidos y competencias de ambas asignaturas. Tal y como recomienda el aprendizaje basado en proyectos, el trabajo se debe finalizar con un producto final, en este caso la realización de dos trabajos de investigación, cada uno de los cuales siguiendo un tipo de metodología de investigación (cuantitativa y cualitativa), los cuales han versado sobre los temas propios de la asignatura «Fundamentos Psicopedagógicos de la Atención a la Diversidad». La exposición de los trabajos se ha concretado en una Jornada Científica sobre Atención a la Diversidad en Educación (ver Figura 1).

Figura 1.

Infografia informativa para el alumnado sobre la práctica interdisciplinar 

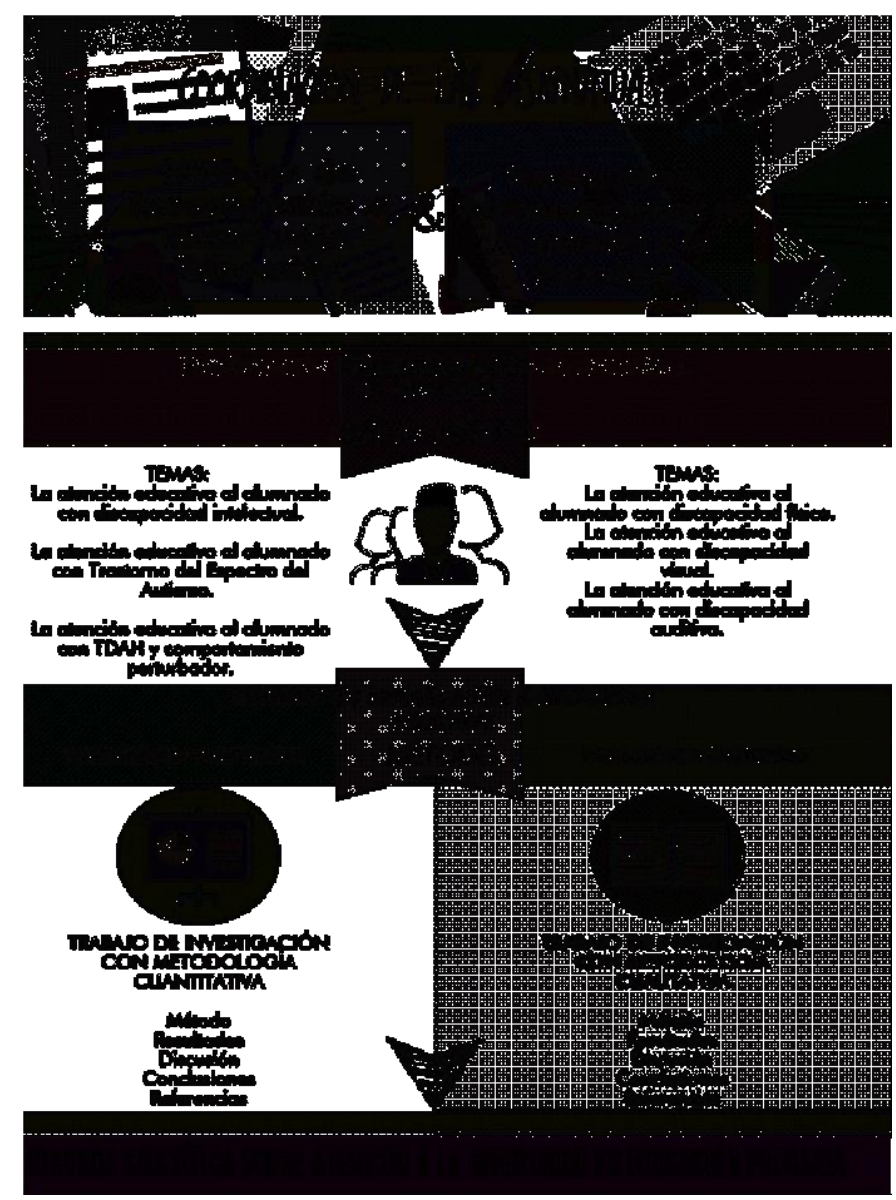

Fuente: elaboración propia.

La práctica interdisciplinar se desarrolló durante 5 meses y se articuló en cinco fases (ver Tabla 1)

Tabla 1.

Temporalización y fases de la práctica interdisciplinar

\begin{tabular}{cl} 
Temporalización & \multicolumn{1}{c}{ Fases de la práctica interdisciplinar } \\
\hline Mes 1 & Identificación de competencias comunes y contenidos a trabajar. \\
\hline Mes 1 & Planificación de la actividad por el equipo docente. \\
\hline Mes 2, 3, 4 y 5 & $\begin{array}{l}\text { Realización de la práctica interdisciplinar de forma coordinada siguiendo la planifi- } \\
\text { cación prevista. }\end{array}$ \\
\hline Mes 5 & $\begin{array}{l}\text { Exposición de los trabajos (Jornada científica de atención a la diversidad en Edu- } \\
\text { cación). }\end{array}$ \\
\hline Mes 5 & $\begin{array}{l}\text { Evaluación de los trabajos (Coevaluación entre alumnado y heteroevaluación por } \\
\text { parte de los docentes). }\end{array}$ \\
\hline Mes 5 & $\begin{array}{l}\text { Cuestionario de evaluación del alumnado participante sobre los resultados obteni- } \\
\text { dos en la práctica interdisciplinar. }\end{array}$ \\
\hline Mes 5 & $\begin{array}{l}\text { Valoración del profesorado implicado en la práctica interdisciplinar a través de en- } \\
\text { trevistas. }\end{array}$ \\
\hline
\end{tabular}

Fuente: elaboración propia (2019).

\subsection{Instrumentos}

La toma de datos se realizó mediante una metodología mixta (cuantitativa y cualitativa). Así, la valoración de la experiencia del alumnado se realizó a través de un cuestionario semiestructurado elaborado ad hoc para esta investigación (ver Tabla 2). El instrumento consta de cuatro partes diferenciadas 
que recogen los siguientes datos: (1) Sociodemográficos: donde se recogieron datos como la edad, el género, el grado en el que estaban matriculados y el número total de asignaturas en las que se habían matriculado; (2) Interés general de la experiencia: en este apartado se recogieron datos relativos a la utilidad percibida de la práctica, la motivación y el interés que suscitaba, la percepción de mejora en la comprensión de los contenidos a través de la práctica y la mejora del trabajo en grupo; (3) Coordinación docente: en este apartado se recogieron datos relativos a la adecuación de la coordinación del profesorado, y a si esta facilitaba el aprendizaje. También se recogieron datos relativos a la comprensión del objetivo de la práctica interdisciplinar y la adecuación percibida del seguimiento coordinado de la práctica interdisciplinar; y (4) Desarrollo y aprendizaje de la práctica: en esta última parte se recogieron datos relativos a adecuación de la documentación, organización y secuenciación de la práctica. Además, se preguntó si la práctica interdisciplinar favoreció la comprensión y aprendizaje de los contenidos de ambas asignaturas. Al final contaban con una pregunta abierta sobre observaciones donde cada estudiante podía escribir cualquier apreciación sobre la actividad de coordinación y colaboración docente llevada a cabo. El instrumento se podía completar en aproximadamente 5 minutos.

Tabla 2.

Cuestionario ad hoc utilizado para la recogida de datos de los estudiantes

A continuación encontrará usted una serie de preguntas, forman parte de la evaluación de un Proyecto de Innovación Docente de la Universidad de Valladolid titulado «Coordinación y colaboración docente en la enseñanza universitaria».

Por razones de confidencialidad, se reservarán los datos personales de los encuestados, al amparo de la Ley Orgánica 15/1999, de 13 de diciembre, de protección de datos de carácter personal (B.O.E. núm. 298, de 14 de diciembre de 1999). Los datos que en él se reflejen serán tratados de manera totalmente CONFIDENCIAL, analizados estadísticamente conjuntamente con el resto de los datos procedentes de la muestra empleada en el estudio y utilizados con los fines propios del trabajo de investigación en el que queda enmarcado el propio cuestionario.

\begin{tabular}{ll}
\hline 1. Edad: & 2. Género: Hombre $\square$ Mujer $\square$ \\
\hline 3. Grado: Primaria $\square$ Titulación Conjunta $\square$ & $\begin{array}{l}\text { 4. Número de asignaturas en las que te has matricu- } \\
\text { lado: }\end{array}$
\end{tabular}

Durante este curso académico (2015-2016) se ba llevado a cabo una experiencia de colaboración entre las asignaturas de «Fundamentos Psicopedagógicos de Atención a la Diversidad» y «Métodos de Investigación e Innovación educativa». A continuación le mostramos una serie de afirmaciones para que exprese su grado de acuerdo o desacuerdo con el fin de evaluar esta experiencia, donde (1) Nada de acuerdo y (4) Totalmente de acuerdo:

\section{INTERÉS GENERAL DE LA EXPERIENCA}

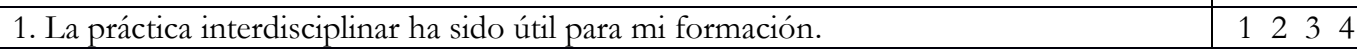

2. La práctica interdisciplinar ha aumentado mi motivación e interés por las asignaturas. $1 \begin{array}{llll}12 & 234\end{array}$

3. La práctica interdisciplinar me ha facilitado el estudio y la comprensión de los con- $\mid \begin{array}{llll}1234\end{array}$ ceptos de ambas asignaturas.

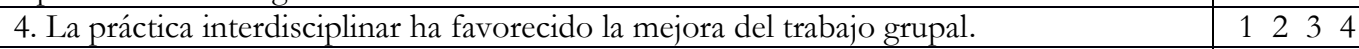

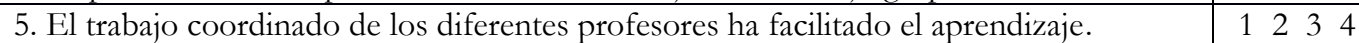

\begin{tabular}{l|l} 
6. Los docentes han trabajado de manera coordinada. & 1234 \\
\hline
\end{tabular}

\begin{tabular}{l|lll} 
7. He entendido el objetivo de la práctica interdisciplinar. & 123 & 2 & 4
\end{tabular}

\begin{tabular}{l|l|l} 
8. El seguimiento de los trabajos por parte de los docentes ha sido adecuado. & 1234
\end{tabular}

\section{DESARROLLO Y APRENDIZAJE EN LA PRÁCTICA}

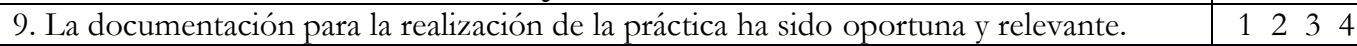

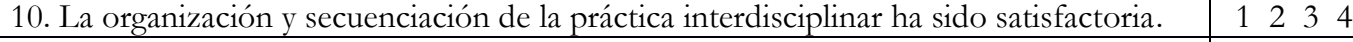

11. Utilizar los temas de la asignatura de «Fundamentos psicopedagógicos de atención a $1 \begin{array}{llll}1 & 2 & 3 & 4\end{array}$ la diversidad» para el trabajo de investigación me ha ayudado a ver la importancia de la investigación en la práctica educativa.

12. Realizar el trabajo de investigación (tanto cualitativo como cuantitativo) ha favorecido la comprensión de los contenidos de la asignatura de «Fundamentos psicopedagógicos de atención a la diversidad».

Observaciones. Por favor, comente todo aquello que considere de interés en relación a la experiencia de colaboración entre las asignaturas de «Fundamentos Psicopedagógicos de Atención a la Diversidad» y «Métodos de Investigación e Innovación educativa».

Fuente: elaboración propia (2018) 


\subsection{Análisis de datos}

El análisis de datos se llevó a cabo con el programa informático SPSS v.20. Se realizó un análisis de tipo descriptivo de cada una de las preguntas del cuestionario, a partir de las principales categorías de interés en la investigación. A partir de ahí, se ha realizado una triangulación de los resultados obtenidos (profesorado y alumnado). Finalmente, se realizaron análisis comparativos en base al género y el grado en el que se encuentra matriculado el estudiante. No se realizaron análisis del contenido de la pregunta abierta puesto que no fue contestada por el alumnado.

\section{Resultados}

\subsection{Valoración de la práctica interdisciplinar: Percepción del alumnado}

\section{Interés general de la práctica interdisciplinar}

En esta primera parte del cuestionario se evaluó la percepción del alumnado sobre aspectos generales de la práctica interdisciplinar, como su utilidad, la motivación y el interés que suscitaba, la comprensión de contenidos de las dos asignaturas implicadas y la mejora del trabajo grupal. Se encontró que un $78.6 \%$ se encontraban bastante o muy de acuerdo con que este tipo de prácticas interdisciplinares son útiles para su formación (ver Figura 2); mientras que un 64 \% se mostró bastante o muy de acuerdo con que la práctica interdisciplinar llevada a cabo había aumentado su motivación e interés por las asignaturas (ver Figura 3).

Figura 2.

La práctica interdisciplinar ha sido útil para mi formación (Item 1)

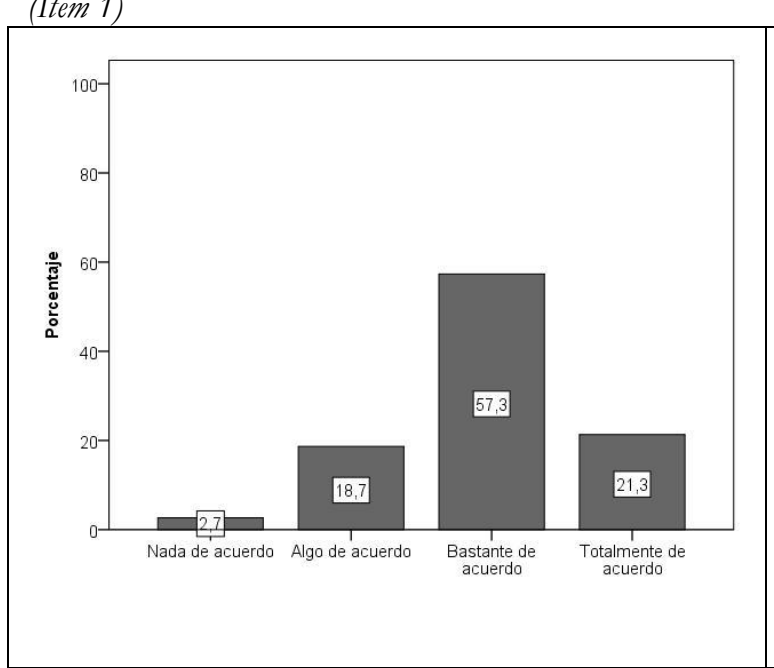

Figura 3.

La práctica interdisciplinar ba aumentado mi motivación e interés por las asignaturas (Item 2)

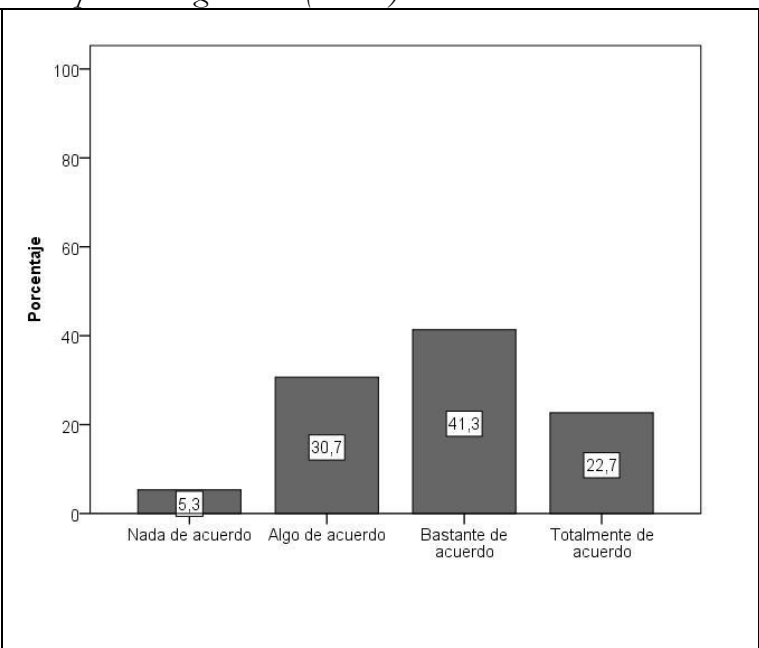

En relación a si la actividad interdisciplinar había facilitado la comprensión de los conceptos de ambas asignaturas, un $76 \%$ se mostró bastante o muy de acuerdo con esta afirmación (ver Figura 4); mientras que un $56 \%$ se consideró bastante o muy de acuerdo con que esta práctica había mejorado su trabajo grupal (ver Figura 5).

Figura 4.

Figura 5. 
La práctica interdisciplinar me ha facilitado el estudio y la comprensión de los conceptos de ambas asignaturas (Item 3)

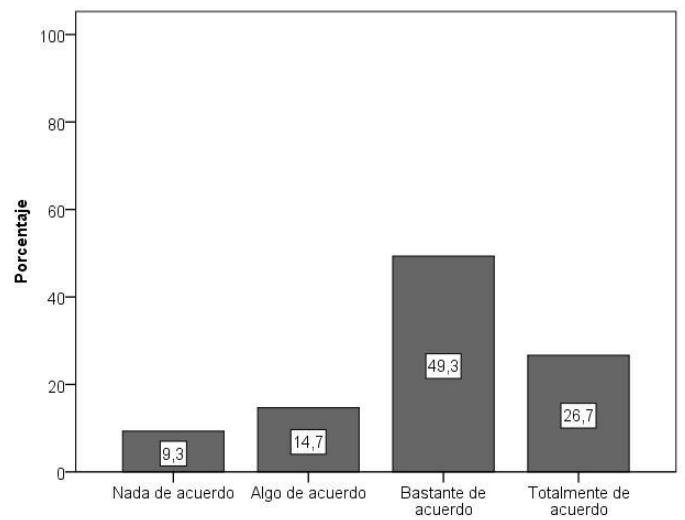

La práctica interdisciplinar ha favorecido la mejora del trabajo grupal (Item 4)

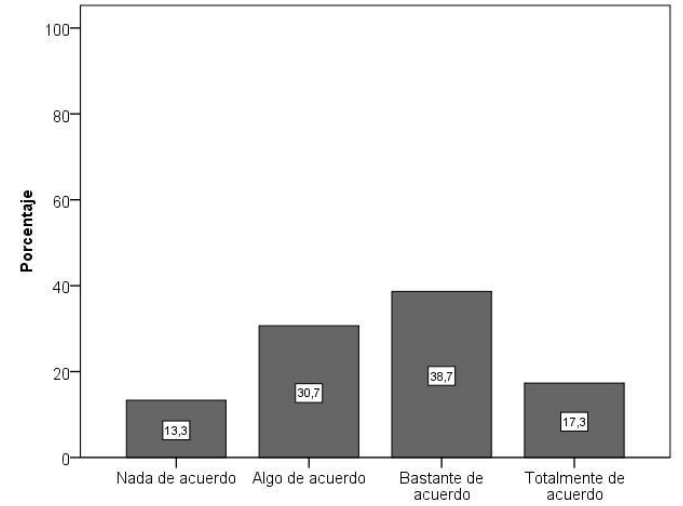

Se llevó a cabo una prueba t de Student para comparar las medias de las respuestas en cada uno de los cuatro ítems en función del género y del Grado en el que los estudiantes se encontraban matriculados y no se encontraron diferencias significativas en ninguno de los ítems.

\section{Coordinación docente en el desarrollo de la práctica interdisciplinar}

En esta segunda parte del cuestionario se evaluó la percepción del alumnado sobre la coordinación que había llevado a cabo el profesorado implicado en la práctica interdisciplinar. Se encontró que un $54.7 \%$ estaban bastante o muy de acuerdo con que la coordinación del profesorado había facilitado el aprendizaje (ver Figura 6); mientras que un $56 \%$ se mostró bastante o muy de acuerdo con que los docentes habían trabajado de forma coordinada (ver Figura 7).

Figura 6.

El trabajo coordinado de los diferentes profesores ha facilitado el aprendizaje

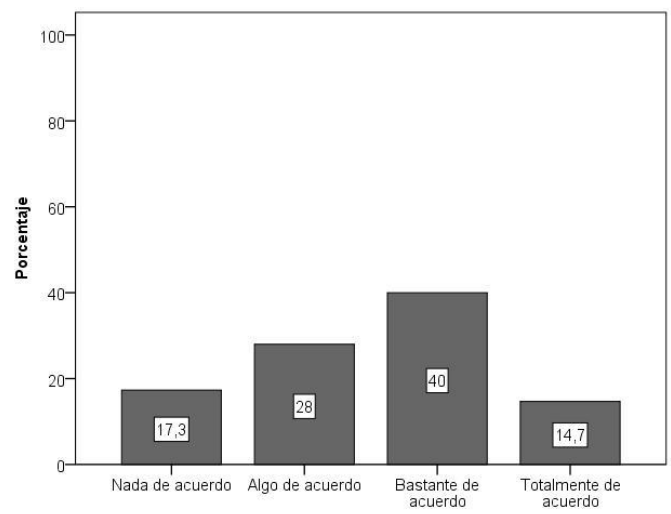

Figura 7.

Los docentes han trabajado de manera coordinada

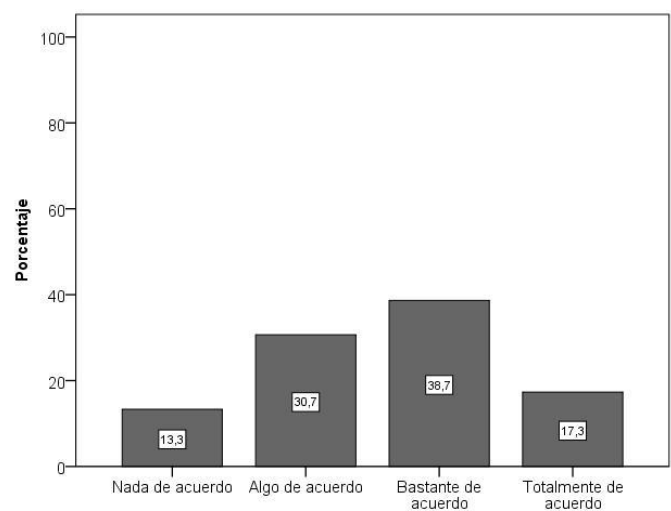

En relación a la comprensión del objetivo de la práctica interdisciplinar, se encontró que $75.7 \%$ del alumnado se mostraba bastante o muy de acuerdo con esta afirmación (ver Figura 8); mientras que un $86.7 \%$ se mostró bastante o muy de acuerdo con que el seguimiento de los trabajos realizado por los docentes había sido el adecuado (ver Figura 9). 
Figura 8.

He entendido el objetivo de la práctica interdisciplinar

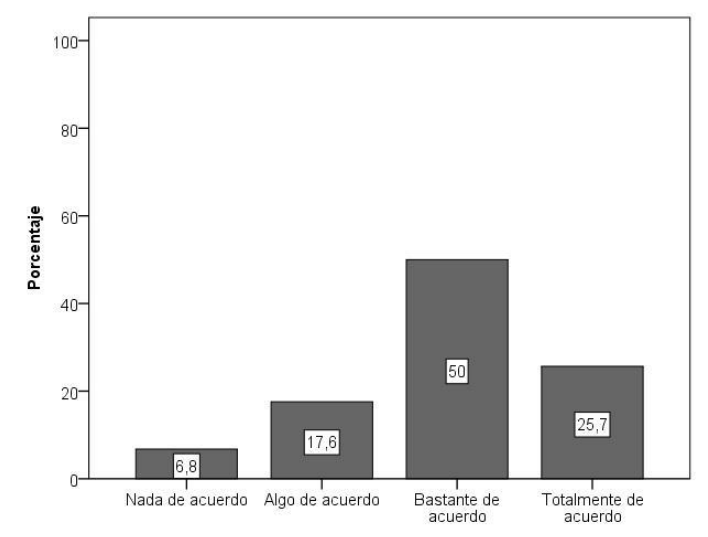

Figura 9.

El seguimiento de los trabajos por parte de los docentes ha sido adecuado

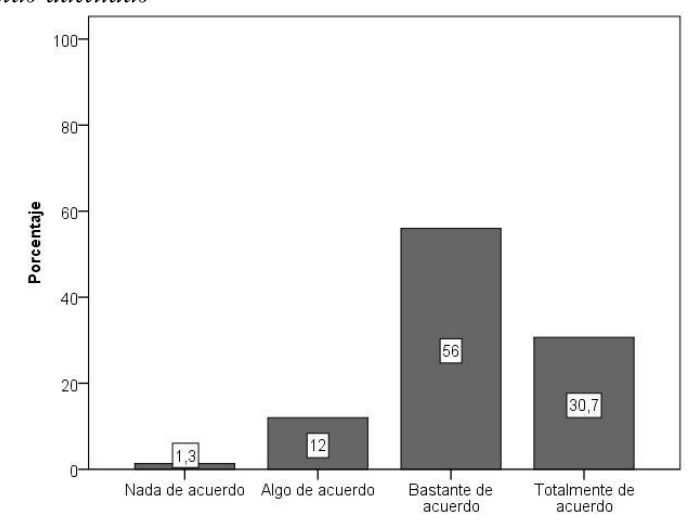

Se llevó a cabo una prueba t de Student para comparar las medias de las respuestas en cada uno de estos cuatro ítems en función del género y se encontraron diferencias significativas en los ítems 5, 6 y 7 , de manera que los varones consideraron en mayor medida que las mujeres que la coordinación docente había mejorado el aprendizaje $(\mathrm{t}(44.11)=3.86, \mathrm{p}=.001)$, que había existido una adecuada coordinación docente $(\mathrm{t}(72)=2.42, \mathrm{p}=.02)$ y que habían entendido el objetivo de la práctica interdisciplinar $(\mathrm{t}(71)=2.24, \mathrm{p}=.03)$ (ver Tabla 3$)$.

Tabla 3.

Estadísticos descriptivos de los items 5, 6 y 7 en función del género

\begin{tabular}{cllll} 
& Género & $\mathrm{N}$ & Media & Desviación típica \\
\hline \multirow{2}{*}{ Ítem 5 } & Hombre & 21 & 3.10 & .77 \\
\cline { 2 - 5 } & Mujer & 53 & 2.28 & .93 \\
\hline \multirow{2}{*}{ Ítem 6 } & Hombre & 21 & 3.00 & .84 \\
\cline { 2 - 5 } & Mujer & 53 & 2.43 & .93 \\
\hline \multirow{2}{*}{ Ítem 7 } & Hombre & 21 & 3.30 & .72 \\
\cline { 2 - 5 } & Mujer & 53 & 2.81 & .86 \\
\hline
\end{tabular}

Fuente: elaboración propia (2019).

Se realizó otra prueba t de Student para comparar las medias de las respuestas en cada uno de estos cuatro ítems en función del grado en el que los estudiantes estaban matriculados y se encontraron diferencias significativas en los ítems 5, 6, 7 y 8, de manera que los estudiantes del Grado de Educación Primaria consideraron en mayor medida que los del Grado de Estudios Conjuntos que la coordinación docente había mejorado el aprendizaje $(\mathrm{t}(73)=4.60, \mathrm{p}=.001)$, que había existido una adecuada coordinación docente $(\mathrm{t}(73)=4.62, \mathrm{p}=.001)$, que habían entendido el objetivo de la práctica interdisciplinar $(\mathrm{t}(72)=2.03, \mathrm{p}=.05)$ y que se había realizado un adecuado seguimiento de los trabajos por parte de los docentes $(\mathrm{t}(73)=3.46, \mathrm{p}=.001)$ (ver Tabla 4$)$.

Tabla 4.

Estadísticos descriptivos de los items 5, 6, 7 y 8 en función del grado 


\begin{tabular}{cllll} 
& \multicolumn{1}{c}{ Género } & $\mathrm{N}$ & Media & Desviación típica \\
\hline \multirow{2}{*}{ Ítem 5 } & Educación Primaria & 41 & 2.93 & .82 \\
\cline { 2 - 5 } & Grado Conjunto & 34 & 2.03 & .87 \\
\hline \multirow{2}{*}{ Ítem 6 } & Educación Primaria & 41 & 3.00 & .81 \\
\cline { 2 - 5 } & Grado Conjunto & 34 & 2.12 & .84 \\
\hline \multirow{2}{*}{ Ítem 7 } & Educación Primaria & 41 & 3.13 & .85 \\
\cline { 2 - 5 } & Grado Conjunto & 34 & 2.74 & .79 \\
\hline \multirow{2}{*}{ Ítem 8 } & Educación Primaria & 41 & 3.39 & .69 \\
& Grado Conjunto & 34 & 2.88 & \\
\hline
\end{tabular}

Fuente: elaboración propia (2019).

\section{Desarrollo y aprendizaje de la práctica interdisciplinar}

Finalmente, en la tercera parte del cuestionario se evaluó la percepción del alumnado sobre el desarrollo de la práctica interdisciplinar y el aprendizaje llevado a cabo en la misma. Se encontró que un $81.3 \%$ se encontraban bastante o muy de acuerdo con que la documentación entregada para realizar la práctica había sido adecuada (ver Figura 10), mientras que un $68 \%$ se mostró bastante o muy de acuerdo con que la organización y secuenciación de la práctica interdisciplinar había sido satisfactoria (ver Figura 11).

Figura 10.

La documentación para la realización de la práctica ha sido oportuna y relevante

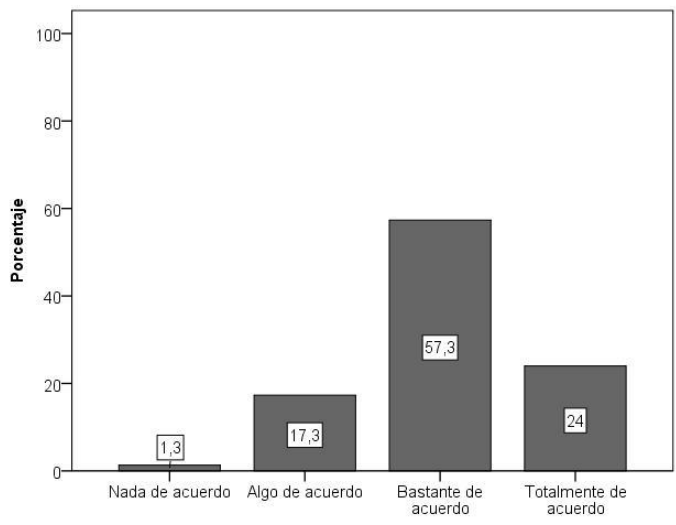

Figura 11.

La organización y secuenciación de la práctica interdisciplinar ha sido satisfactoria

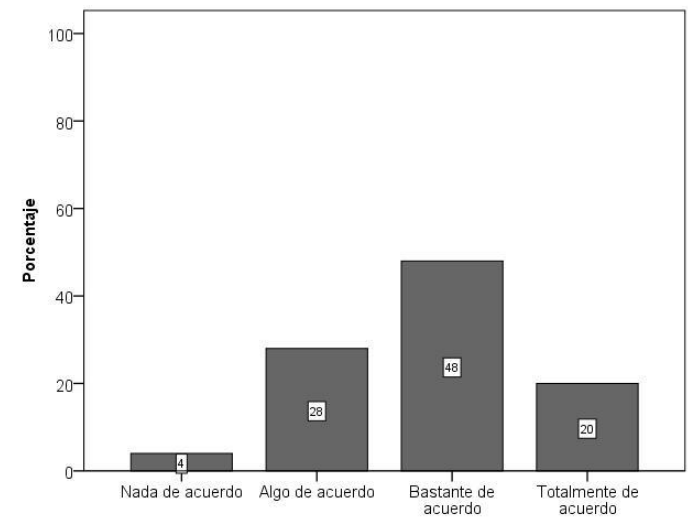

En relación al aprendizaje y comprensión de contenidos de las asignaturas implicadas, se encontró que un $82.2 \%$ del alumnado se mostró bastante o muy de acuerdo con que la utilización de los temas de la asignatura de «Fundamentos psicopedagógicos de atención a la diversidad» les había ayudado a comprender la importancia de la investigación en la práctica educativa (ver Figura 12). Mientras que un $71.6 \%$ se mostró bastante o muy de acuerdo con que la realización de la práctica interdisciplinar en investigación había favorecido la comprensión de los contenidos de la asignatura de «Fundamentos psicopedagógicos de atención a la diversidad» (ver Figura 13).

Figura 12.

Utilizar los temas de la asignatura de «Fundamentos psicopedagógicos de atención a la diversidad» me ha ayudado a ver la importancia de la investigación en la práctica educativa
Figura 13.

Realizar la práctica interdisciplinar en investigación ha favorecido la comprensión de los contenidos de la asignatura de «Fundamentos psicopedagógicos de atención a la diversidad» 

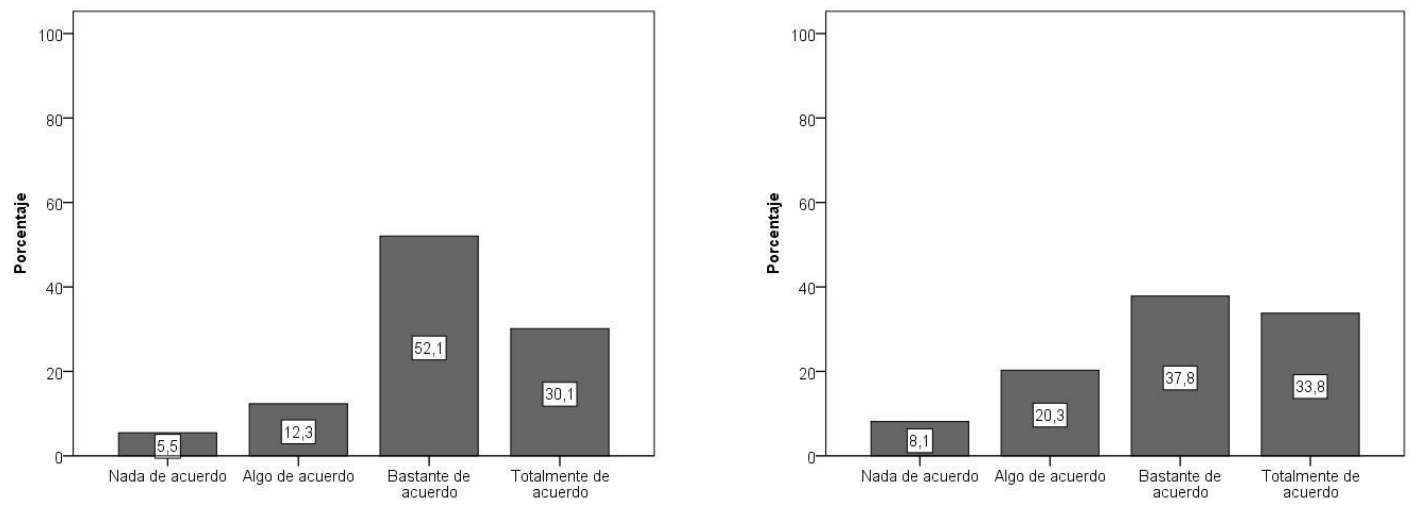

Se llevó a cabo una prueba t de Student para comparar las medias de las respuestas en cada uno de estos cuatro ítems en función del género y se encontraron diferencias significativas en el ítem 9, de manera que los varones consideraron en mayor medida que las mujeres que la documentación para la realización de la práctica había sido oportuna y relevante $(\mathrm{t}(72)=3.23, \mathrm{p}=.002)$ (ver Tabla 5).

Tabla 5.

Estadísticos descriptivos del item 9 en función del género

\begin{tabular}{cllcc} 
& Género & $\mathrm{N}$ & Media & Desviación típica \\
\hline \multirow{2}{*}{ Ítem 9} & Hombre & 21 & 3.43 & .60 \\
\cline { 2 - 5 } & Mujer & 53 & 2.89 & .67 \\
\hline
\end{tabular}

Fuente: elaboración propia (2019).

Se llevó a cabo otra prueba t de Student para comparar las medias de las respuestas en cada uno de estos cuatro ítems en función del grado en el que los estudiantes estaban matriculados y se encontraron también diferencias significativas en los ítems 9 y 10, de manera que los estudiantes del Grado de Educación Primaria consideraron en mayor medida que los del Grado de Estudios Conjuntos que la documentación para la realización de la práctica había sido oportuna y relevante $(\mathrm{t}(73)=2.20, \mathrm{p}=.03)$ y la organización y secuenciación de la práctica había sido satisfactoria $(\mathrm{t}(73)=2.29, \mathrm{p}=.03)$ (ver Tabla 6).

Tabla 6.

Estadísticos descriptivos de los items 9 y 10 en función del Grado

\begin{tabular}{cllll} 
& Género & $\mathrm{N}$ & Media & Desviación típica \\
\hline \multirow{2}{*}{ Ítem 9} & Educación Primaria & 41 & 3.20 & .68 \\
\cline { 2 - 5 } & Grado Conjunto & 34 & 2.85 & .67 \\
\hline \multirow{2}{*}{ Ítem 10 } & Educación Primaria & 41 & 3.02 & .79 \\
\cline { 2 - 5 } & Grado Conjunto & 34 & 2.62 & .74 \\
\hline
\end{tabular}

Fuente: elaboración propia (2019).

\section{Valoración de la práctica interdisciplinar: Percepción del profesorado}

En cuanto a la valoración de la práctica interdisciplinar por parte del profesorado, se realizó un análisis de contenido de las entrevistas realizadas al final de la experiencia. Las categorías analizadas fueron:

\section{Utilidad de la experiencia en tu práctica profesional}

Se valora la experiencia como interesante y se reconoce que ha habido un aprendizaje de las distintas metodologías y estrategias de enseñanza utilizadas por los otros compañeros. 
El proceso de coordinación para el desarrollo de la experiencia es valorado por todo el profesorado de manera positiva, aunque se reconocen las dificultades para llevarlo a cabo: ausencia de momentos específicos de coordinación, falta de horas para llevar esas reuniones a cabo:

«La coordinación pues ha sido buena... Claro, a costa como siempre de nuestros tiempos personales, aunque hubiera sido deseable contar con más tiempo para revisar y planificar las acciones conjuntas. Lo que sí que es verdad es que planificar una asignatura así, te supone el doble de trabajo que de la otra forma (Profesora 1).»

\section{Evaluación del desarrollo de la práctica}

«La organización, secuenciación y documentación ha sido adecuada, pero es necesario pedir a los estudiantes entregas periódicas del trabajo realizado porque si no es así lo dejan todo para el final y no pueden desarrollar correctamente el trabajo, duplicándose en muchos casos la asistencia a las tutorías (Profesora 2).»

\section{Fortalezas y debilidades de estas prácticas}

Entre los aspectos positivos de la práctica profesional se reconoce su utilidad para un aprendizaje interdisciplinar que conecte distintas asignaturas, conectando contenidos y generando una visión más globalizada del conocimiento. Otro aspecto señalado en las entrevistas es la posibilidad de aprender de los compañeros.

Como aspectos negativos se insiste en que ha supuesto un mayor trabajo por parte del profesorado y les ha obligado a invertir tiempo personal en la coordinación. El alumnado, como norma general, está muy poco acostumbrado a estas prácticas y a planificar de manera sistemática su trabajo:

«Al dejar el trabajo para el final no iban entendiendo bien los conceptos que se trabajaban en clase y por tanto al final tenían tantas dudas que estaban superados. En este momento han colapsado las horas de tutorías de las profesoras. Otra cuestión importante es la ausencia de valoración por parte de la administración de estas experiencias (Profesora 3).»

\section{Discusión y conclusiones}

No es fácil desarrollar la colaboración entre docentes, y aun cuando el profesorado trabaja de forma colectiva no siempre sale bien y supone un intercambio sustancial sobre los objetivos de la educación, los problemas de la práctica o las demandas de aprendizaje. En una obra ya clásica, Sarason (1982) explica que hace falta mucha observación, discusión y comunicación en las culturas escolares para reflexionar sobre los comportamientos profesionales y, si procede, cambiarlos.

Aquí presentamos los resultados del trabajo de coordinación que el profesorado implicado ha realizado a través de una práctica interdisciplinar en el Grado de Educación de la Universidad de Valladolid. Esta experiencia ha supuesto una mayor carga de trabajo para el profesorado implicado ya que a las tareas habituales del docente se han añadido las de organización conjunta de la práctica, comunicación continua durante el proceso y consenso en la evaluación de la misma. Pero, a la luz de los datos proporcionados por nuestro alumnado, consideramos que ha sido una experiencia positiva, porque, en general, creemos que ha mejorado el aprendizaje y la calidad del mismo en ambas asignaturas. La mayoría de nuestro alumnado considera que prácticas interdisciplinares como la llevada a cabo son útiles y mejoran la comprensión de los contenidos académicos.

La coordinación docente supone un trabajo intenso, donde es fundamental encontrar vías de comunicación rápidas, flexibles y continuas, porque si esto no se cumple este trabajo estará abocado al fracaso. Creemos que encontrar espacios y vías de comunicación que tengan las características ya citadas ha sido una de las debilidades en nuestra práctica interdisciplinar. $Y$ así parece que lo percibe nuestro alumnado, ya que únicamente un $56 \%$ considera que la coordinación ha sido adecuada. Sin embargo, se han valorado muy positivamente los seguimientos que se han realizado de los proyectos de investigación, quizá tener varios profesores a los que acudir para realizar un seguimiento de la práctica facilita que ellos perciban que se está tutorizando de forma adecuada su realización. Esto implica, en algunas ocasiones, una duplicidad en los esfuerzos del profesorado, ya que un mismo 
trabajo es evaluado por varios profesores en diferentes momentos, pero también enriquece al alumnado, ya que recibe información sobre su desempeño desde diferentes puntos de vista. También han sido muy positivas el resto de valoraciones relativas al desarrollo de la práctica interdisciplinar, de manera que la mayoría del alumnado considera que la documentación proporcionada ha sido adecuada, la organización y secuenciación de la práctica también y que el carácter interdisciplinario de la práctica ha mejorado la comprensión de los contenidos de ambas asignaturas.

Por tanto, encontramos ventajas y desventajas en la realización de este tipo de prácticas aportadas en la triangulación de los datos aportados por profesores y alumnos. Las ventajas afectan fundamentalmente al alumnado ya que, por una parte, parece que mejora su comprensión y aprendizaje de los contenidos de las asignaturas implicadas y, por otra, disminuye su carga de trabajo. Para el profesorado, por el contrario, aumenta el número de tareas a realizar, ya que debe coordinarse y comunicarse con sus compañeros habitualmente, en la línea de lo apuntado por Gallego, Redondo, Lorente y Benedito (2011), pero también supone un aprendizaje continuo de nuevas metodologías, formas de resolución de problemas..., de manera que mejora su práctica docente.

La investigación sobre el trabajo colaborativo resulta estratégica para cambiar y mejorar nuestro sistema educativo y está en la base de cualquier proceso de innovación educativa (Donmoyer, YennieDonmoyer y Galloway, 2012; Sebastian y Allensworth, 2012; Vescio, Ross y Adams, 2008). La colaboración puede resultar estratégica para el docente en la medida en que lo nutra de nuevas herramientas pedagógicas ante los problemas concretos de su práctica, reforzando así su autonomía y capacidad de decisión. Por ello, los docentes necesitan trabajar en entornos colaborativos donde abunde el apoyo mutuo, las responsabilidades compartidas y la reflexión sistemática. Sin embargo, no podemos dejar de tener en cuenta las dificultades que se deben afrontar en este camino, por lo que se aconseja diseñar y planificar cuidadosamente las actividades a realizar y recurrir a las nuevas tecnologías que favorecen la comunicación rápida, flexible y fluida que requiere este tipo de experiencias (p. ej. campus virtuales, listas de distribución, etc.).

Creemos que es necesario seguir investigando sobre las prácticas colaborativas del profesorado e incorporar en ellas la voz de los alumnos, ya que son un factor clave del cambio educativo y de mejora de los centros educativos y contribuyen, en definitiva, a una mejor calidad de la educación.

\section{Referencias}

Aranda, L. (2015). Un acercamiento al aprendizaje colaborativo en educación superior. El aprendizaje colaborativo al alcance de todos. Relingüistica aplicada, 16.

Benito, A., \& Cruz, A. (2005). Nuevas claves para la docencia universitaria en el Espacio Europeo de Educación Superior. Madrid: Narcea.

Berzosa, I.; \& Arroyo, M.J. (2014). El aprendizaje basado en problemas en las áreas de ciencias: un ejemplo en la asignatura «Ciencias para el mundo contemporáneo». En II Congreso Virtual Internacional sobre Innovación Pedagógica y Praxis Educativa INNOV AGOGÍA 2014 (521-528).

Cáceres, P., \& García, E. (2005). La acción tutorial en la universidad. Servei de formació permanent: Universitat de València.

Carreras, J., \& Perrenoud, P. (2005). El debat sobre les competències a l'ensenyament universitari. Barcelona: Universitat de Barcelona.

Cuadrado, M., Ruiz, M. E., \& Coca, M. (2009). Participación y rendimiento del estudiante universitario en un proyecto docente interdisciplinar, bilingüe y virtual. Revista de Educación, 348, pp. 505518.

Donmoyer, R., Yennie-Donmoyer, J., \& Galloway, F. (2012). The search for connections across principal preparation, principal performance, and student achievement in an exemplary principal preparation program. Journal of Research on Leadership Education, 7, pp. 5-43. doi: $10.1177 / 1942775112440631$

Durall, E., \& Leinonen, T. (2014). Pensamiento de diseño y aprendizaje colaborativo. Comunicar: Revista cientifica iberoamericana de comunicación y educación, 42, pp. 107-116. doi: 10.3916/C42-2014-10

Gairín, J. (2000). Cambio de cultura y organizaciones que aprenden. Educar, 27, pp. 31-85. doi: $10.5565 /$ rev/educar.245 
Gallego, J., Redondo, A., Lorente, R., \& Benedito, A. (2011). La coordinación entre profesores como base del nuevo aprendizaje universitario. Arxius, 24, pp. 119-134.

Hargreaves, A., \& Shirley, D. (2012). The Global Fourth Way. The Quest for Educational Excellent. Thousand Oaks, CA: Corwin Press.

Jarauta, B. (2014). El aprendizaje colaborativo en la universidad: referentes y práctica. REDU: Revista de Docencia Universitaria, 12(4), pp. 302. doi: 10.4995/redu.2014.5624

Jiménez, M. Á. S., \& Coronil, A. G. (2018). Desarrollo de un equipo docente en la coordinación del profesorado en el ámbito universitario. Alteridad, 14(1), pp. 98-108. doi: 10.17163/alt.v14n1.2019.08

Johnson, D.W., Johnson, R.T., \& Holubec, E.J. (1999). El aprendizaje cooperativo en el aula. Buenos Aires: Paidós.

Johnson, D. W., \& Johnson, R. T. (2014). La evaluación en el aprendizaje cooperativo: cómo mejorar la evaluación individual a través del grupo. Madrid: SM.

Krichesky, G.J., \&Murillo F. J. (2015). La colaboración docente como factor de aprendizaje y promotor de mejora. Un estudio de casos. Educación XX1.

Learreta, B. (2006). La coordinación del profesorado ante las demandas del espacio europeo de educación superior: el caso de la Facultad de CAFYD en la UEM. Madrid: Comunicación Gráfica S.L.

Little, J., \& Horn, I. (2007). 'Normalizing' problems of practice: converting routine conversation into a resource for learning in professional communities. En L. Stoll, \& K.S. Louis (Eds.) Professional Learning Communities: Divergence, Depth and Dilemmas (pp. 79-92). Columbus: Open University Press.

López-Pastor, V. M., \& Pérez-Puello, Á. (2017). Buenas prácticas docentes. Evaluación formativa y compartida en educación: Experiencias de éxito en todas las etapas educativas. León: Universidad de León.

Martínez, M., \& Viader, M. (2008). Reflexiones sobre aprendizaje y docencia en el actual contexto universitario. La promoción de equipos docentes. Revista de Educación, no extraordinario, pp. 213234.

McLaughlin, M., \& Talbert, J. (2006). Building School-Based Teacher Learning Communities. Professional strategies to improve student achievement. Nueva York: Teacher's College Press.

Morales, L. (2007). La integración lingüística del alumnado inmigrante. Madrid: CIDE.

Navarro, I., \& López, B. (2019). Aprendizaje cooperativo basado en proyectos y entornos virtuales para la formación de futuros maestros. Educar, 55(2), pp.1-23. doi: 10.5565/rev/educar.935

OCDE (2003). La definición y selección de competencias clave. Resumen ejecutivo. Recuperado de http://www.deseco.admin.ch/bfs/deseco/en/index.

Palmer, A., Montaño, J.J., \& Palou, M. (2009). Las competencias genéricas en la educación superior. Estudio entre la opinión de empleadores y académicos. Psicothema, 21(3), pp. 433- 438.

Peñaloza, J. (2017). Incidencia del aprendizaje colaborativo en la práctica educativa. Didácticas Específicas, 16, pp. 46-60.

Pérez, A. Y Soto, E. (2011). Lesson Study. La mejora de la práctica y la investigación docente. En Cuadernos de Pedagogía, no 417, pp. 64-67.

Pinedo, R., Caballero, C., \& Fernández, A.M. (2016). Metodologías activas y aprendizaje por competencias en las enseñanzas de grado. En J. Castejón (Ed.) Psicología y Educación: Presente y Futuro (pp. 448-457). Madrid: ACIPE.

Pou, R. (2009). El desarrollo de proyectos interdisciplinarios. En Seminario de reflexión RED-U-USC: La coordinación mediante equipos docentes en ES: fortalezas, recursos y necesidades. Recuperado de http://congresos.um.es/redu/2-09/paper/view/1661/1631.

Sánchez-Cantalejo, Y., Rodríguez Jiménez, R. M., Sáez Pizarro, B., Velasco Quintana, P.J., \& Fernández Prieto, M. (2011). Transversalidad y coordinación de profesorado de ciencias: una experiencia en los nuevos grados. En Actas de las VII Jornadas Internacionales de Innovación Universitaria. Villaviciosa de Odón: Universidad Europea de Madrid.

Scott, C.L. (2015). El futuro del aprendizaje 2, ¿Qué tipo de aprendizaje se necesita en el siglo XXI? Investigación y Prospectiva en Educación, 14.

Sebastian, J., \& Allensworth, E. (2012). The influence of principal leadership on classroom instruction and student learning: A study of mediated pathways to learning. Educational Administration Quarterly, 48, pp. 626-663. doi: 10.1177/0013161X11436273

Senge, P. (2002). La Quinta Disciplina: Escuelas que Aprenden. Bogotá: Norma. 
Simón, C., Echeita, G., \& Sandoval, M. (2018). La incorporación de la voz del alumnado a la 'Lesson Study' como estrategia de formación docente y mejora para la inclusión. Cultura y Educación, 30, pp. 215-225. doi: 10.1080/11356405.2017.1416741

Smith, S. C., \& Scott, J. J. (1990). The collaborative school: a work environment for effective instruction. Eugene: University of Oregon.

Terrón, M.J. (2009). 2 a 2: una propuesta para facilitar la coordinación docente y el aprendizaje. En Jornada de experiencias de coordinación por medio de equipos docentes en la enseñanza universitaria. Universitat de València.

Torrego, L., \& Ruiz, C. (2011). La coordinación docente en la implantación de los títulos de Grado. Revista Electrónica Interuniversitaria de Formación Del Profesorado REIFOP, 14(4), pp. 31-40.

Vescio, V., Ross, D., \& Adams, A. (2008). A review of research on the impact of professional learning communities on teaching practice and student learning. Teaching and Teacher Education, 24(1), pp. 80-91. doi: 10.1016/j.tate.2007.01.004

Zabalza, M.A. (Comp.) (2006). La Universidad y la docencia en el mundo de hoy. Bogotá: Pontificia Universidad Javeriana. 